\title{
Numerical Simulations on Aerodynamic Characteristics of a Guided Rocket Projectile
}

\author{
Jiazhong Peng ${ }^{1, a}$, Liangyu Zhao ${ }^{1,2, b}$, Longyin Jiao ${ }^{1, c}$ \\ ${ }^{1}$ School of Aerospace Engineering, Beijing Institute of Technology, Beijing 100081, P. R. China; \\ ${ }^{2}$ Key Laboratory of Dynamics and Control of Flight Vehicle, Beijing Institute of Technology, \\ Beijing 100081, P. R. China. \\ apengjiazhong86@163.com, ${ }^{\mathrm{b}}$ zhaoly@bit.edu.cn, 'Iongyin113@126.com
}

Keywords: Aerodynamics characteristics, guided rocket projectile, CFD.

\begin{abstract}
Aerodynamic characteristics of a guided rocket projectile are numerically simulated. Simulations are performed in a range of flight conditions using a commercial CFD software FLUENT, and variations of lift coefficients and drag coefficients with Mach numbers and angles of attack were demonstrated. The numerical results also indicate that the angle of canard deflection has significant influence on the flow field near tailfins.
\end{abstract}

\section{Introduction}

Aerodynamic characteristics of a guided projectile are necessary to predict its trajectory and design its guidance control system. Normally, three kinds of approaches are available to get the aerodynamic characteristics: theoretical analysis, wind tunnel test and computational fluid dynamics (CFD).

Since 1970s, the CFD method had been used firstly, it is becoming a common way to solve fluid dynamics problems [1]. As it can obtain simulation results in relatively shorter time with less cost, the CFD method has been widely employed in the design stage of a flight vehicle. Besides, the CFD results contain abundant date which can be used to investigate complex physical phenomena, such as the flow interactions, pressure distribution and turbulent features. DeSpirito and Heavey calculated Magnus moment and roll damping of a projectile with CFD method, the results match the experimental data well [2]. Yue and Chang compute the aerodynamic characteristics of an air-to-air missile with CFD method [3]. Li simulated a missile which flies in the ground effect area based on CFD method, and studied the aerodynamics and flight mechanics of the missile [4]. Nelson and McGowan researched the flow interaction and rolling moment model of Sparrow missile by analyzing CFD results [5]. With the development of computer technologies, complex problems can be calculated more accurately using CFD method and many intricate problems can be studied more detailed. As a result, the design cycle can be shortened and design efficiency can be improved. Coyle and Silton applied CFD method to investigate the aerodynamic characterization of a high maneuverability projectile [6]. Sahu and Fresconi carried through a CFD-based simulation of the stability a projectile's canards at high angle of attack and the interaction of these canard vortices on the afterbody-fins [7].

\section{Computational Simulation}

The governing equations of CFD are the Navier-Stokes equations, and the vector form of the Navier-Stokes equations is shown as:

$$
p \frac{\mathrm{d} \boldsymbol{v}}{\mathrm{d} t}=-\nabla p+\rho \boldsymbol{F}+\mu \Delta \boldsymbol{v}
$$

where $p$ is the pressure, $\boldsymbol{v}$ is the velocity, $\rho$ is the density, $\boldsymbol{F}$ is the volume force and $\mu$ is the dynamic viscosity. In principle, the Navier-Stokes equations describe both laminar and turbulent flows without the need of additional information. However, the direct numerical simulation of the turbulent flows at realistic Reynolds numbers would consume significant computational cost. 
Therefore, researchers usually employ Reynolds-Averaged Navier-Stokes (RANS) equations to describe turbulent flows. In consequence of Reynolds stresses were added into RANS equations, and proper turbulence model is needed to closure the equations.

The geometric model of the guided rocket projectile analyzed in this paper is displayed in Fig. 1. It is comprises of three components: the cylindrical body with a tapered nose, four flat plate canards and six flat plate tailfins arranged evenly on the body. The rocket projectile has a large fineness ratio and the value of $l / d$ is about 25 (where $l$ is the length and $d$ is the diameter of the projectile).

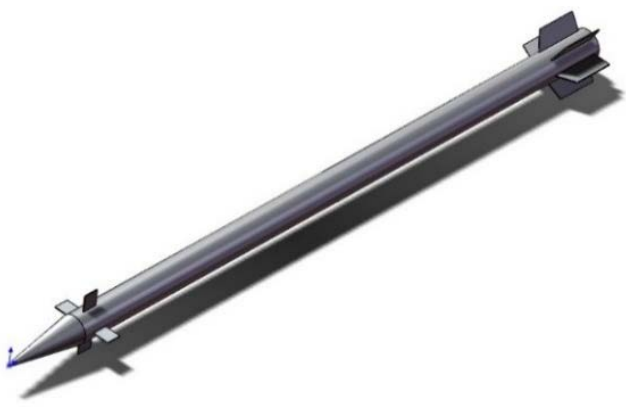

(a) 3-D model

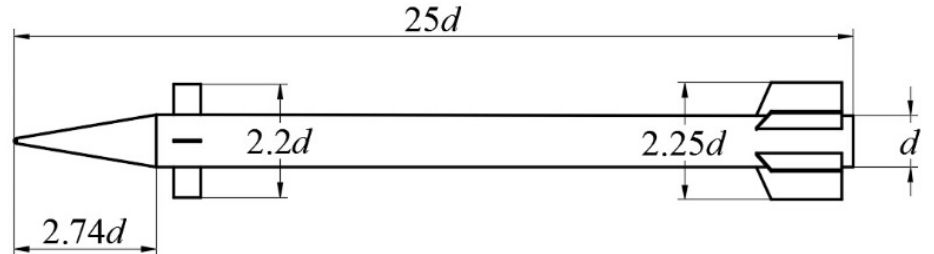

(b) Top view

Fig. 1 Geometric model of the guided projectile

The numerical mesh used for this investigation is shown in Fig. 2 and the computational domain consists of 2673370 hexahedral cells. In order to satisfy the subsonic, transonic and supersonic cases, there is a large domain around the projectile. The upstream boundary of the domain is $2 l$ far from the nose, the downstream boundary is $3 \mathrm{l}$ far from the tail of the projectile, and the diameter of the domain is $25 \mathrm{~d}$. In the process of mesh generation, the full computational domain was split into $8 \times 7 \times 10$ zones, and then configure the grid number of each zone. The computational mesh presented here was created by the commercial mesh generation tool ANSYS ICEM CFD 15.0. It is a flexible mesh generation tool and can offer the capability to parametrically create volume or surface meshes from geometry or meshes in multi-block structured or unstructured formats.

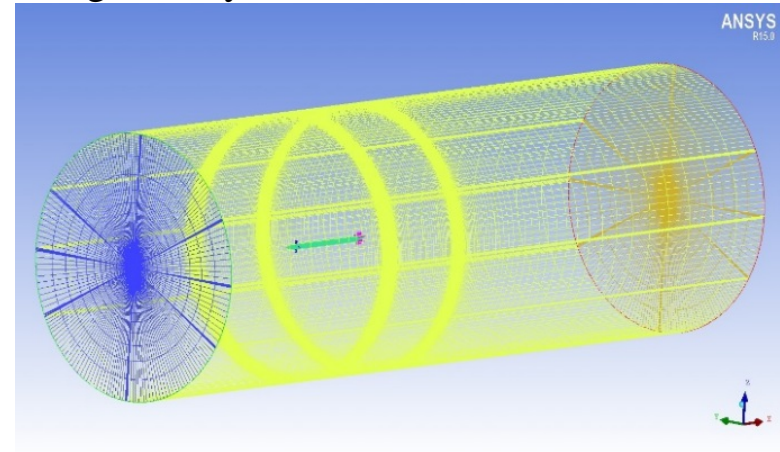

(a) Full computational domain

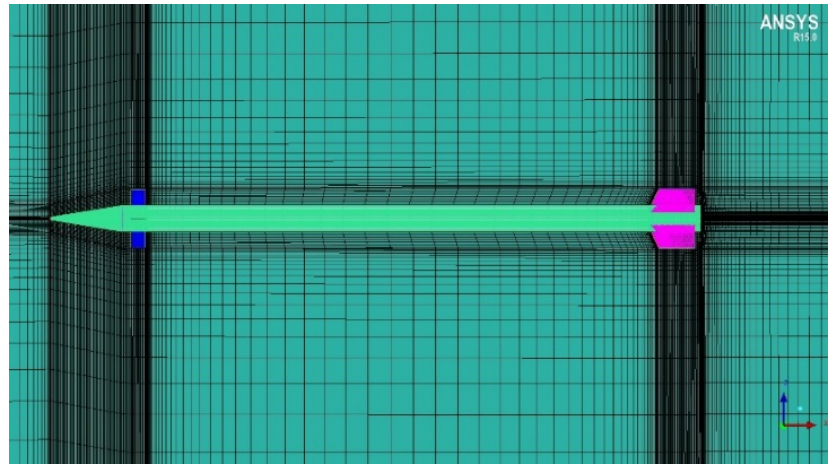

(b) Grids near the rocket body

Fig. 2 Numerical grid for the investigation

Fig. 2 (a) displays the full computational domain for this simulation. Fig. 2 (b) shows the details of the numerical grid near the rocket body. There is a very high cell density region near the surface of the rocket projectile, which is helpful to get more accurate result in the concerned particular zone.

The commercial CFD software FLUENT 15.0 by ANSYS Inc. was used for the simulations. In order to obtain the flow solution, FLUENT is used to numerically solve the 3-D, compressible, steady-state RANS equations. And the two equations SST $k-\omega$ turbulence model is chosen to closure the RANS equations. For solving these equations, density-based implicit solver provided by FLUENT is selected. The coupled implicit approach is employed to solve for all variables in all cells at the same time, and as a result it has higher accuracy and better astringency.

Flight condition was discretized into numbers of flight statuses, and every status of them need to be simulated. In the process of trajectory prediction, the continuous flight condition can be obtained by interpolating the CFD results. The discretization of angle of attack, Mach number and angle of 
canard deflection is shown in Table 1 , and totally $6 \times 10 \times 3$ flight statuses were computed. Yaw and roll movement of the rocket projectile were not considered in the simulation.

Table 1 Discretization of flight conditions

\begin{tabular}{cc}
\hline Item & Value \\
\hline Angle of attack & $0^{\circ}, 2^{\circ}, 4^{\circ}, 6^{\circ}, 8^{\circ}, 10^{\circ}$ \\
Mach number & $0.6,0.8,1.0,1.2,1.5,2.0,2.5,3.0,3.5,4.0$ \\
Angle of canard deflection & $0^{\circ}, 5^{\circ}, 10^{\circ}$ \\
\hline
\end{tabular}

\section{Result and Discussion}

The lift coefficients and the drag coefficients change with different angles of attack under different Mach numbers are shown in Fig. 3 and Fig. 4. As shown in Fig. 3, the lift coefficient increases with the increase of the Mach number in the subsonic stage and decreases with the increase of the Mach number in the supersonic stage, and approach to the maximum value near 1.0 Mach. The curves rise rapidly from 0.6 Mach to 1.0 Mach and decrease quickly from 1.0 Mach to 1.5 Mach, and then change smoothly with the increase of Mach number. The relationship between drag coefficient and Mach numbers is similar with lift coefficient as shown in Fig. 4. It should be mentioned that, although the force coefficient is decreasing in the supersonic zone, the force is growing with the increase of Mach number, as the force is proportional to the square of the velocity.

It is also observed in Fig.3 and Fig.4 that both the lift coefficient and the drag coefficient are going up with the increase of the angles of attack at every Mach number. Because the shape of the rocket projectile is axial symmetry, the lift coefficient at $0^{\circ}$ attack angle is zero as displayed in Fig.3. The growth rate of the lift coefficient with the increase of angle of attack is approximately constant, while the drag coefficient does not. From Fig.4, it clearly shows that the drag coefficient rises slowly at small angle of attack and become quickly with the enlargement of angle of attack.

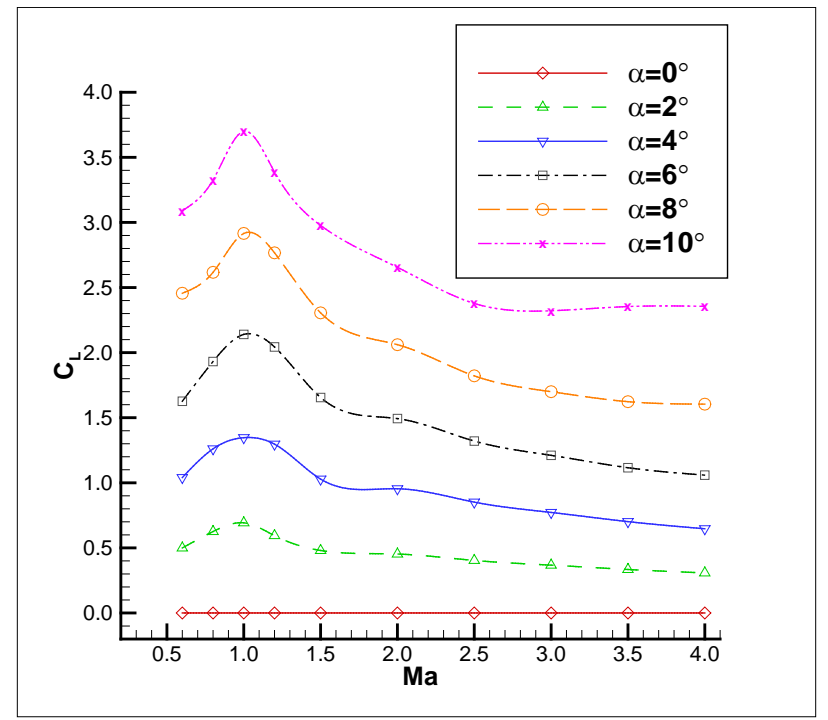

Fig. 3 Curves of the lift coefficient changing with the Mach numbers

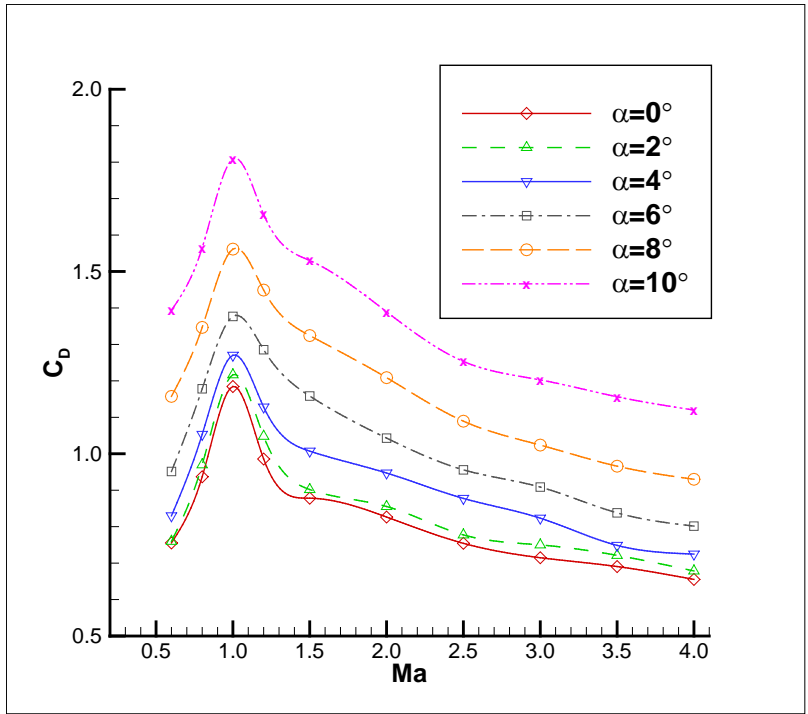

Fig.4 Curves of the drag coefficient changing with the Mach numbers

Fig. 5 displays streamlines produced by the simulations under $6^{\circ}$ angle of attack and 3.0 Mach with three angles of canard deflection. It can be seen from the three graphs that the vortices developed at the root of the canards continue to travel downstream to the location of the tailfins, but which at the tip does not. When the angle of canard deflection is $0^{\circ}$, as it is shown in Fig. 5 (a), the most streamlines go to the tailfins area when they passed from the canard area. And, with the angle of canard deflection increasing, as we can see from Fig. 5 (b) and Fig. 5 (c), fewer of the streamlines cross the tailfins area when they have went through the canard area. However, the fewer streamlines have greater effect. It is observed by the color of the streamlines that, with the increase of the angle of canard deflection, the velocity variation of the fluid in the tailfins area is greater. The 
phenomenon indicates that the vortices in the area are more excited and significant. Therefore, the flow interference between canards and tailfins becomes serious with the increase of angle of canard deflection.

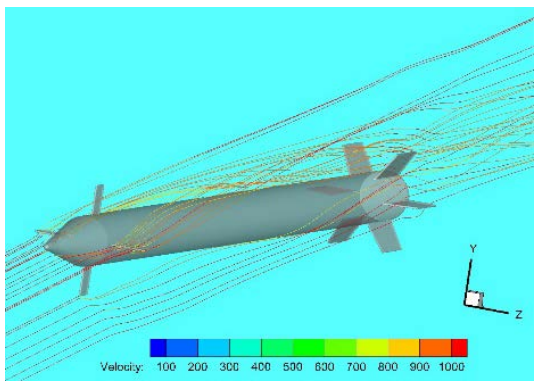

(a) $0^{\circ}$ canard deflection

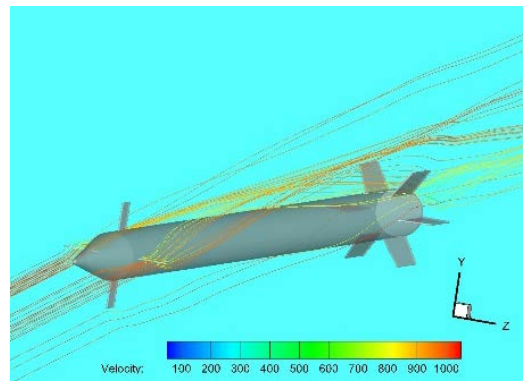

(b) $5^{\circ}$ canard deflection

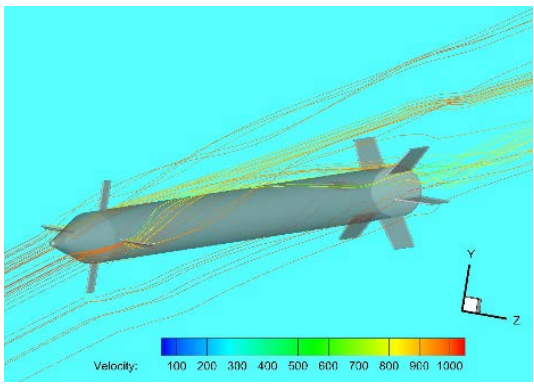

(c) $10^{\circ}$ canard deflection

Fig. 5 Streamlines with $2^{\circ}$ and $10^{\circ}$ angle of attack under 3.0 Mach

\section{Summary}

Numerical simulations on the aerodynamic characteristic of a large fineness ratio guided rocket projectile using CFD were carried out. The CFD results indicate that the lift coefficient and drag coefficient have the similar variation trend with the Mach numbers, and both of them change rapidly in the transport area but smoothly in subsonic and supersonic area. It is also observed that the two force coefficients rise with the increase of the angle of attack, but the variation of the rising speed is different. From the interaction analysis, it is concluded that the canard deflection has much to do with the flow field of the tailfins. The vortices developed at root of the canards have greater effect than the tip of the canards. The flow interaction between canards and tailfins become serious with the increase of the canard deflection angle.

\section{Acknowledgement}

The grant support from the National Science Foundation of China (No. 11202023) is greatly acknowledged.

\section{References}

[1] J.D. Anderson, Computational Fluid Dynamics: The Basic with Application, McGraw-Hill, Inc., New York, 1995.

[2] J. DeSpirito, K.R. Heavey, CFD Computation of Magnus Moment and Roll-Damping Moment of a Spinning Projectile, AIAA Atmospheric Flight Mechanics Conference and Exhibit, American Institute of Aeronautics and Astronautics, 2004.

[3] C.G. Yue, X.L. Chang, S.J. Yang and Y.H. Zhang, Numerical Simulation on Aerodynamic Characteristic of an Air-to-Air Missile, Computer Science for Environmental Engineering and Ecoinformatics. Springer Berlin Heidelberg, 2011: 472-476.

[4] F. Li, H. Yang, W. Shi and Z.G. Li, Investigation of Aerodynamics and Flight Mechanics Characteristics of the Ground Effect Missile, 52nd Aerospace Sciences Meeting, 2014.

[5] A.C. Nelson, G.Z. McGowan and F.G. Moore, CFD Database for the Development of a Non-Linear Missile Rolling Moment Model, 53rd AIAA Aerospace Sciences Meeting, 2015.

[6] C.J. Coyle, S.I. Silton, CFD Aerodynamic Characterization of a High Maneuverability Airframe, 33rd AIAA Applied Aerodynamics Conference. 2015: 3015.

[7] J. Sahu, F. Fresconi, Flight Behaviors of a Complex Projectile using a Coupled CFD-based Simulation Technique: Free Motion, 33rd AIAA Applied Aerodynamics Conference. American Institute of Aeronautics and Astronautics, 2015. 